\title{
New Methods for Structural and Functional Analysis of Complex Processes
}

\author{
Miguel Castaño Arranz ${ }^{\dagger \star}$ and Wolfgang Birk $^{\dagger}$
}

\begin{abstract}
The selection of the structure of a controller in large scale industry processes requires extensive process knowledge. The aim of this paper is to present methods which help designers to comprehend a process by representing structural and functional relationships from actuators and process disturbances to measured or estimated variables. This paper describes similarities between brain connectivity theory and interaction analysis in multivariable processes. Results from both theories are combined to create new methods for the analysis of industry processes. The developed methods are applied to an illustrative example.
\end{abstract}

\section{INTRODUCTION}

$\mathbf{P}$ ROCESS industries have to operate in a very competitive and globalized environment, requiring efficient and sustainable production processes. Production targets need to be translated into control objectives and are usually formulated as performance specifications of the process, i.e. tracking of references or rejection of process disturbances [1]. This is often a hard and difficult task which involves assumptions and simplifications because of the process complexity. Complexity arises often due to the large scale character of a process, i.e. large number of manipulated and controlled variables. A critical step is then the choice of the structure of the control, which means that controllers need to be placed between sensors and actuators. Often the control structures are sparse, which means that the information of only a reduced set of sensors is used to manipulate certain actuators, sets which are reflected by constructions as cascades, feedforward and feedback.

Current methods for control structure design use so called Interaction Measures (IMs), i.e. the Relative Gain Array (RGA) [2], Participation Matrix (PM) [3], Hankel Index Interaction Array (HIIA) [4] or $\Sigma_{2}$ [5]. IMs compare the effect of the manipulated inputs of the process on the controlled variables in order to suggest a structure for the controller.

Control structure design should also take into consideration the rejection of process disturbances. To reject process disturbances the actuators need to be manipulated in order to drive the outputs to the desired operating state. An input energy into the process is then required to achieve this rejection. Comparing the relative effect of the process disturbances and manipulated inputs into the controlled variables would also help to take decisions about the control structure.

Thus control structure design requires extensive knowledge about how the different variables in the process are

\footnotetext{
* Corresponding author: miguel. castanodltu.se.

$\dagger$ Control Engineering Group, Department of Computer Science and Electrical Engineering, Luleå University of Technology, SE-971 87 Luleå, Sweden.
}

interconnected, that is, how is the flow of energy propagated through the process. Such analysis would allow designers to comprehend this flow, and thereby the cause/effect relationship in the process. In [6], structural matrices had been used to obtain qualitative information about these interconnections in order to determine feasible control structures.

Previous work on analysis of structural and functional relationships in industrial processes use the coherence analysis described by Akaike [7]. In [8] coherence functions are used to identify noise sources and noise propagation in a Boiling Water Reactor. Coherence functions were modified in [9] in order to analyze closed loop processes.

Coherence analysis has also been used the field of brain connectivity. Neurons and neural populations interact with each other in different brain processes related to events as perception, or cognition. Electroencephalography (EEG) is a measure of electrical activity in the brain which is acquired from sensors positioned on the surface of the head, each of the electrodes collects the aggregated voltage of a neuron population. Analyzing the flow of information between populations of neurons allows to understand the communication between different parts of the brain in different brain processes. In a very similar way, analyzing the flow of information between variables in an industrial process will provide designers with the required information to understand the behavior of the plant.

The aims of this paper are:

- introduce some of the relationships between brain connectivity theory and interaction measures,

- combine both theories in order to obtain methods for the analysis of industrial processes which show up causal and functional relationships describing the propagation of energy in the process. This will help designers to formulate control objectives and decide about the control structure.

The paper layout is as follows: Section II gives the preliminaries needed in IMs (Section II-A) and brain connectivity (Section II-B), besides some relationships between both theories are commented in Section II-C, and the previous work in structural analysis of industrial processes is described in Section II-D. The framework of the research conducted in this paper is in this way established. Section III is the main result of this paper, and describes the methodology used for structural (Section III-C) and functional (Section III-D) analysis of industrial processes. An illustrative example is conducted in Section IV: the process subject to analysis is described (Section IV-A), the analysis is performed (Section IV-B) and the results are discussed (Section IV-C). Finally the conclusions are stated in Section V. 


\section{PRELIMINARIES}

\section{A. Interaction Measures and Control Structure Selection}

The basic idea of the IMs is to compare the effect of the manipulated inputs of the process on the controlled variables. Actuators are then grouped with a set of variables (measured by sensors) on which they relatively strongly affect, and the structure of the controller is in this way decided. This is achieved by neglecting dynamic channels in the process which have a smaller significance than a designed threshold. Only a subset of the process dynamics is therefore considered to design the controller.

$\Sigma_{2}$ was introduced in [5] and some of its properties were derived in [10]. For a linear multivariable process described by a transfer function $G_{m \times n}(s)$ the $\Sigma_{2}$ was defined as [5]

$$
\left[\Sigma_{2}\right]_{i j}=\frac{\left\|G_{i j}(s)\right\|_{2}}{\sum_{k l}\left\|G_{k l}(s)\right\|_{2}}
$$

For any of the $G_{i j}(s)$ SISO systems, if $G_{i j}(s)$ is stable and strictly proper, $\left\|G_{i j}(s)\right\|_{2}$ can be expressed as

$$
\left\|G_{i j}(s)\right\|_{2}=\sqrt{\frac{1}{2 \pi} \int_{-\infty}^{\infty}\left|G_{i j}(j \omega)\right|^{2} d w}
$$

It has been shown [10] that the squared $\mathcal{H}_{2}$ norm of each elemental SISO subsystem can be interpreted as the coupling in terms of the energy transmission rate between the past inputs and the current output. Note also that all the elements of $\Sigma_{2}$ add up to 1 . Therefore the larger elements in $\Sigma_{2}$ identify the input-output channels which are able to exchange larger amounts of energy.

\section{B. Partial Directed Coherence (PDC) and Directed Transfer Function (DTF) in Brain Connectivity}

PDC was first introduced in [11] to describe direct flow of effects in multivariate times series modeled as multivariate autoregressive processes (MVAR), and used to reveal the flow of information between the cortex and hippocampus during a spindle episode in the slow-wave sleep, in which $12-16 \mathrm{~Hz}$ waves are propagated through the brain to inhibit other brain processes and keep the sleeper in a tranquil state.

PDC is estimated from EEG measured signals, and assumes that a MVAR model of the process is available:

$$
\left(\begin{array}{c}
x_{1}(n) \\
\vdots \\
x_{N}(n)
\end{array}\right)=\sum_{r=1}^{p} A_{r}\left(\begin{array}{c}
x_{1}(n-r) \\
\vdots \\
x_{N}(n-r)
\end{array}\right)+\left(\begin{array}{c}
w_{1}(n) \\
\vdots \\
w_{N}(n)
\end{array}\right)
$$

The frequency dependant matrix $\bar{A}$ is computed as:

$$
\bar{A}(j \omega)=I-\left.\sum_{r=1}^{p} A_{r} \cdot z^{-r}\right|_{z=e^{-j \omega}}
$$

The PDC from $x_{j}$ to $x_{i}$ is

$$
\bar{\pi}_{i j}(j \omega)=\frac{\bar{A}_{i j}(j \omega)}{\sqrt{\sum_{m=1}^{N}\left|\bar{A}_{m j}(j \omega)\right|^{2}}}
$$

The normalization of $\bar{\pi}_{i j}(j \omega)$ is then performed through columns, the element $\bar{\pi}_{i j}(j \omega)$ is different than 0 only if there exists a direct causal connection from $x_{j}$ to $x_{i}$, thus, $\bar{\pi}$ has the same structure as the matrix $\bar{A}(j \omega)$ representing the process, and the element $\bar{\pi}_{i j}(j \omega)$ represents the effect of $x_{j}$ on $x_{i}$ compared to the effect of $x_{j}$ on all the other states which it directly affects. Therefore, the $j^{\text {th }}$ column of $\bar{\pi}(j \omega)$ represents the relative effect from $x_{j}$ to the states of the process directly affected by it.

More recently [12], the sPDC was introduced as:

$$
s P D C_{i j}(\omega)=\frac{\left|\bar{A}_{i j}(j \omega)\right|^{2}}{\sum_{m=1}^{N}\left|\bar{A}_{m j}(j \omega)\right|^{2}}
$$

PDC and SPDC provide direct structural information of the process, that is, if $\bar{A}_{i j}(j \omega)=0$ then $\bar{\pi}_{i j}(j \omega)=0$. However, state $j$ might affect state $i$ through the other states of the process even if $\bar{\pi}_{i j}(j \omega)=0, \forall \omega$. Therefore, there exists a functional relationship between the states, event which is reflected by DTF.

DTF was first introduced in [13] to determine direction and frequency content in the brain activity flow, and also assumes that a MVAR description of the process as in (2) is available. The DTF is then defined as:

$$
\operatorname{DTF}_{i j}(\omega)=\frac{\left|H_{i j}(j \omega)\right|}{\sqrt{\sum_{m=1}^{N}\left|H_{i m}(j \omega)\right|^{2}}}
$$

with $H(j \omega)=(\bar{A}(j \omega))^{-1}$.

If both random processes $\mathrm{x}(\mathrm{t})$ and $\mathrm{w}(\mathrm{t})$ are jointly wide sense stationary, then the power spectral density $S_{x x}(\omega)$ of $x(t)$, can be expressed as:

$$
S_{x x}(\omega)=H(j \omega) \cdot S_{w w}(\omega) \cdot H^{*}(j \omega)
$$

Thus (5) describes how the input power is converted in the process by $H(j \omega)$, and yields the power content of $x(t)$. The normalization applied in (4) means that the sum of all the contributions from the input channels to the $j^{\text {th }}$ output is equal to 1 .

\section{Interaction Measures and Brain Connectivity}

Both brain connectivity theory, and interaction measures share several common features. They try to determine interactions in different processes in order to obtain a proper understanding of the process behavior. Fig. 1 will be used to express the difference between both theories. The figure collects all the possible variables taking part in a process: $u_{1}, \ldots, u_{m}$ represent the manipulated inputs, $x_{1}, \ldots, x_{n}$ are lumped states representing controlled variables, internal states and estimated variables, and $w_{1}, \ldots, w_{v}$ represent $v$ independent sources of process disturbances. An element in the depicted matrix represents the influence of a variable in a lumped state associated with the corresponding column and row respectively.

Some of the brain processes fit an MVAR model, in which no exogenous input excites the process. It is then modeled as 


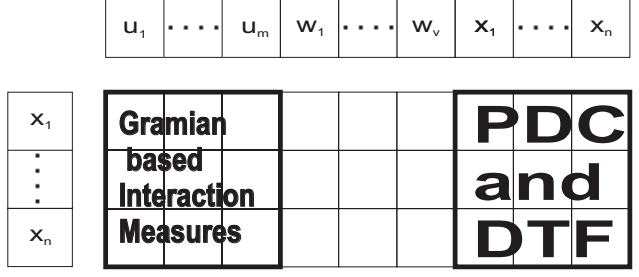

Fig. 1. Possible interconnections in an open-loop process. Relationships analyzed by interaction measures and by brain connectivity.

an autonomous process with white noise input, in which each of the EEG sensors is represented by a state. Therefore, brain connectivity analyzes functional and structural relationships between states in MVAR models.

The IMs used in control theory measure the exchange of energy from the exogenous inputs of the process to the controlled variables, being this exchange of energy carried out through the internal interconnections of the plant. Therefore, IMs provide functional information about the input-output relationship.

We aim to analyze all the effects (structural and functional) represented in Fig. 1 by combining elements from both theories and including in the analysis the propagation of process disturbances.

\section{Structural Matrices}

When the matrix in Fig. 1 is filled with boolean elements representing whether there is a causal dependence of the variables or not, it is denoted structural matrix [6].

Structural matrices have been used in [6] as a tool to determine feasible control structures based on the concept of controllability and observability. Visualization of the cause/effect relationship of the process was also provided by associating structural matrices to digraphs. Further work on structural matrices and digraphs can be consulted in [14] and [15].

Structural matrices are used as a first stage tool to extract information of the plant which helps to decide about the control structure. In section Section III-C we aim to substitute the boolean types in the structural matrices by either a frequency domain description of the relative effect between variables or a real valued weight describing this effect in order to associate the structural matrix with a weighted digraph.

\section{METHOD DESCRIPTION}

\section{A. Process Description}

Assume a linear time invariant process described by a set of differential equations of the form:

$$
\begin{aligned}
& x_{i}^{\left(n_{x_{i}}\right)}=a_{i i 0} x_{i}+a_{i i 1} x_{i}^{\prime}+a_{i i 2} x_{i}^{\prime \prime}+\cdots+a_{i i\left(n_{x_{i}}-1\right)} x_{i}^{\left(n_{x_{i}}-1\right)}+ \\
& \sum_{k=1, k \neq i}^{m}\left(a_{i k 0} x_{k}+a_{i k 1} x_{k}^{\prime}+a_{i k 2} x_{k}^{\prime \prime}+\cdots+\right. \\
& \left.a_{i k\left(n_{x_{j}}-1\right)} x_{i}^{\left(n_{x_{k}}-1\right)}\right)+\sum_{l=1}^{n}\left(b_{i l 0} u_{l}+b_{i l 1} u_{l}^{\prime}+b_{i l 2} u_{l}^{\prime \prime}+\right. \\
& \left.\cdots+b_{i l\left(n_{x_{l}}-1\right)} u_{i}^{\left(n_{u_{l}}-1\right)}\right)+\sum_{t=1}^{v}\left(n_{i t} w_{t}\right)
\end{aligned}
$$

which can be represented as a MVARX process

$$
\begin{aligned}
& \left(\begin{array}{c}
x_{1}^{\left(n_{x_{1}}\right)} \\
x_{2}^{\left(n_{x_{2}}\right)} \\
\vdots \\
x_{n}^{\left(n_{x_{n}}\right)}
\end{array}\right)=\sum_{r=0}^{p-1} A_{r}\left(\begin{array}{c}
x_{1}^{(r)} \\
x_{2}^{(r)} \\
\vdots \\
x_{n}^{(r)}
\end{array}\right)+\sum_{r=0}^{q-1} B_{r}\left(\begin{array}{c}
u_{1}^{(r)} \\
u_{2}^{(r)} \\
\vdots \\
u_{m}^{(r)}
\end{array}\right) \\
& +N\left(\begin{array}{c}
w_{1} \\
w_{2} \\
\vdots \\
w_{v}
\end{array}\right) ; \begin{array}{l}
p=\max \left\{n_{x_{1}}, n_{x_{2}}, \ldots, n_{x_{n}}\right\} \\
q=\max \left\{n_{u_{1}}, n_{u_{2}}, \ldots, n_{u_{m}}\right\}
\end{array}
\end{aligned}
$$

$n$ is the number of states, $m$ is the number of inputs and $v$ is the number of sources of disturbance. $A_{r} \in \mathbb{R}^{n \times n}, B_{r}$ $\in \mathbb{R}^{n \times m}, \mathrm{~N} \in \mathbb{R}^{n \times v}$, the $A_{r}$ and $B_{r}$ matrices are constructed from the differential equations of the process,

$$
A_{r}=\left(\begin{array}{ccc}
a_{11 r} & \ldots & a_{1 n r} \\
a_{21 r} & \ldots & a_{2 n r} \\
\vdots & \ddots & \vdots \\
a_{n 1 r} & \ldots & a_{n n r}
\end{array}\right) ; B_{r}=\left(\begin{array}{ccc}
b_{11 r} & \ldots & b_{1 m r} \\
b_{21 r} & \ldots & b_{2 m r} \\
\vdots & \ddots & \vdots \\
b_{n 1 r} & \ldots & b_{n m r}
\end{array}\right)
$$

Note that the influence of the $n_{x_{i}}^{\text {th }}$ order derivatives into a state can be introduced in (6) using the coefficients of the $A_{r}$ matrices. This means that there is no need to define extra states to represent influence of higher order derivatives. We call these $n$ variables lumped states and we lump in them the internal variables and measured variables which are of interest for the analysis.

Denote the polynomial matrices $A_{f}, B_{f}$ and $A_{\Upsilon}$ as:

$$
\begin{gathered}
A_{f}(s)=\sum_{r=0}^{p-1} A_{r} \cdot s^{r} ; B_{f}(s)=\sum_{r=0}^{q-1} B_{r} \cdot s^{r} \\
A_{\Upsilon}(s)=\operatorname{diag}\left(s^{n_{x_{1}}}, s^{n_{x_{2}}}, \ldots, s^{n_{x_{n}}}\right)-A_{f}(s)
\end{gathered}
$$

\section{B. Normalization}

We will further use the following normalizations.

Given a matrix $G(j \omega) \in C^{m \times n}$, we define:

$$
\left[\Phi_{r}(G(j \omega))\right]_{i j} \triangleq\left\{\begin{array}{cl}
0 & \text {,if } \sum_{l=1}^{n}\left|G_{i l}(j \omega)\right|^{2}=0 \\
\frac{\left|G_{i j}(j \omega)\right|^{2}}{\sum_{l=1}^{n}\left|G_{i l}(j \omega)\right|^{2}} & , \text { otherwise }
\end{array}\right.
$$

A normalization is then applied on the rows of a matrix formed by $\left|G_{i j}(j \omega)\right|^{2}$. When the normalization is applied on the columns, we denote it as $\Phi_{c}(G(j \omega))$.

For a MIMO system represented by $G_{m \times n}(s)$, for which all the elementary subsystems are stable and strictly proper, and dropping the term $s$ for the sake of simplicity, we define:

$$
\left[s \Sigma_{2, r}(G)\right]_{i j} \triangleq\left\{\begin{array}{cl}
0 & , \text { if } \sum_{l=1}^{n}\left\|G_{i l}\right\|_{2}=0 \\
\frac{\left(\left\|G_{i j}\right\|_{2}\right)^{2}}{\sum_{l=1}^{n}\left(\left\|G_{i l}\right\|_{2}\right)^{2}} & , \text { otherwise }
\end{array}\right.
$$


A normalization is then applied on the rows of a matrix formed by $\left(\left\|G_{i j}\right\|_{2}\right)^{2}$. When the normalization is applied on the columns, we denote it as $s \Sigma_{2, c}(G)$.

Normalizations $\Phi_{r}$ and $\Phi_{c}$ yield to frequency dependant matrices, and will be used to obtain a measure of the strength of the process interconnections in frequency domain. Note that these normalizations are very similar to the ones performed by DTF and SPDC in (4) and (3).

Normalizations $s \Sigma_{2, r}$ and $s \Sigma_{2, c}$ yield real valued matrices, and will be used to obtain an overall measure of the strength of the process interconnections. Note that these normalizations are similar to versions of the $\Sigma_{2}$ in which $\left\|G_{i j}\right\|_{2}$ has been squared, and the normalizing has been applied to the rows $\left(s \Sigma_{2, r}\right)$ or the columns $\left(s \Sigma_{2, c}\right)$.

Note that these normalizations involve that the rows of $\Phi_{r}(G)$ and $s \Sigma_{2, r}(G)$ either add up to 1 or all the elements of the row are 0 , whereas the columns of $\Phi_{c}(G)$ and $s \Sigma_{2, c}(G)$ either add up to 1 or all the elements of the column are 0 . This means that $\Phi_{r}$ compares the elements of the same row of $\left[\left|G_{i j}\right|^{2}\right]$ in frequency domain, whilst $s \Sigma_{2, r}$ compares the squared $\mathcal{H}_{2}$ norm of the elements in the same row of $G$. In a similar way the comparisons of $\Phi_{c}$ and $s \Sigma_{2, c}$ are performed through the columns.

\section{Structural Analysis of a process}

Now we provide a description of the methodology used to obtain the structural information of the plant.

$$
a \Upsilon(s) \triangleq\left[-B_{f}(s)|-N \quad| \quad A_{\Upsilon}(s)\right]
$$

Rows and columns of $a \Upsilon(s)$ relate the same variables as the matrix in Fig. 1. Hence $\Phi_{r}(a \Upsilon(j \omega))$ gives direct information on the structure of the plant. We define the Structural Dynamic Power Transfer $(S D P T)$ as:

$$
\operatorname{SDPT}(\omega) \triangleq \Phi_{r}(a \Upsilon(j \omega))
$$

The element $S D P T_{i j}$ represents the influence of the past values of the variable represented in column $\mathrm{j}$ into the future values of $x_{i}$ compared to the influence of the rest of the variables which are directly affecting $x_{i}$. The element $S D P T_{i(i+m+v)}$, which is relating $x_{i}$ with itself represents the own dynamics of the lumped state $x_{i}$, that is, how the past values of $x_{i}$ couple to the future ones compared to the coupling from the other variables which are directly affecting to $x_{i}$. SDPT can be seen as a continuous time version of sPDC in which the exogenous input to the process, and the process disturbances are included as time series which participates in the flow of energy through the process.

$S D P T$ is a frequency dependant matrix; to obtain an overall measure which compresses the frequency domain information and shows causal relationships in the process the $\mathcal{H}_{2}$ norm will be used. The fact that $a \Upsilon(s)$ includes nonproper transfer functions implies that $\mathcal{H}_{2}$ norm is not defined for some of the elements of $a \Upsilon(s)$. Therefore matrix $\Upsilon(s)$ is defined,

$$
[\Upsilon(s)]_{i j} \triangleq\left\{\begin{aligned}
0 & , \text { if } i+m+v=j \\
\frac{-[a \Upsilon(s)]_{i j}}{[a \Upsilon(s)]_{i(i+m+v)}} & , \text { otherwise. }
\end{aligned}\right.
$$

Assuming that each of the elements in $\Upsilon(s)$ is stable and strictly proper, we can compute $s \Sigma_{2, r}(\Upsilon(s))$, and thereby determine weights in a directed graph which represents the structure of the plant. $s \Sigma_{2, r}(\Upsilon(s))$ will be named Structural Energy Transfer (SET).

$$
S E T \triangleq s \Sigma_{2, r}(\Upsilon(s))
$$

\section{Functional Analysis of a process}

Matrix $a \Upsilon(s)$ is constructed based on the direct causality of the plant, that is, it has the same structure as $\left[B_{f}(s)|N| A_{f}(s)\right]$, and therefore the same structure as the plant.

However, the proposed method for structural analysis gives limited information about the following questions:

- how is the effect in one of the manipulated inputs propagated through the rest of the process?,

- how is a process disturbance propagated through the process?,

- how is a process variable affected by the manipulated inputs and process disturbances?,

- how is the interaction between the internal variables of the process?.

To answer these questions, we will provide in this section methods to perform a numerical analysis and visual representation of these effects.

The autoregressive part of the process and the effect of the external inputs are separately analyzed.

1) Effect of the process inputs: Let's define matrix $\Omega$ as

$$
\Omega(s) \triangleq\left[A_{\Upsilon}^{-1}(s) B_{f}(s) \quad \mid \quad A_{\Upsilon}^{-1}(s) N\right]
$$

Matrices $\Phi_{c}(\Omega(j \omega))$ and $\Phi_{r}(\Omega(j \omega))$ are proposed as a frequency domain description of how the inputs into the process (manipulated variables and process disturbances) are affecting to the lumped states. They will be named Functional Dynamic Power Transfer $(F D P T)$ :

$$
F D P T_{r}(\omega) \triangleq \Phi_{r}(\Omega(j \omega)) \quad ; \quad F D P T_{c}(\omega) \triangleq \Phi_{c}(\Omega(j \omega))
$$

Each of the first $m$ columns of $F D P T_{c}$ represents the relative effect of a manipulated variable $u_{i}$, with $i=\{1, \ldots, m\}$ onto the rest of the process, and the remaining $v$ columns represent how each of the process disturbances $w_{i}$, with $i=\{1, \ldots, v\}$ is propagated through the rest of the process.

Each of the $n$ columns of $F D P T_{r}$ represent the relative effect of the manipulated variables of the process and the process disturbances into the $n^{\text {th }}$ lumped state.

When an overall measure is to be obtained representing the relative effects in the process with a real-valued matrix in order to obtain the weights for a digraph, $s \Sigma_{2, r}(\Omega(s))$ and $s \Sigma_{2, c}(\Omega(s))$ are computed, and named Functional Energy Transfer (FET):

$$
F E T_{r} \triangleq s \Sigma_{2, r}(\Omega(s)) \quad ; \quad F E T_{c} \triangleq s \Sigma_{2, c}(\Omega(s))
$$


2) Interactions in the autoregressive part of the process: The interactions between states are obtained using the concept of DTF. Assume that in the process described by (6), the exogenous input and the process disturbances combined are a random wide sense stationary processes $\xi=\left(\xi_{1}, \ldots, \xi_{n}\right)^{T}$, affecting the process as in (9).

$$
\begin{aligned}
\left(\begin{array}{c}
x_{1}^{\left(n_{x_{1}}\right)} \\
\vdots \\
x_{n}^{\left(n_{x_{n}}\right)}
\end{array}\right) & =\sum_{r=0}^{p-1} A_{r}\left(\begin{array}{c}
x_{1}^{(r)} \\
\vdots \\
x_{n}^{(r)}
\end{array}\right)+\left(\begin{array}{c}
\xi_{1} \\
\vdots \\
\xi_{n}
\end{array}\right) \\
p & =\max \left\{n_{x_{1}}, n_{x_{2}}, \ldots, n_{x_{n}}\right\}
\end{aligned}
$$

$\xi$ is then considered to be the input of the process, the output $x$ of the linear process is also a wide sense stationary process, and $\xi$ and $x$ are jointly wide sense stationary processes. Then, the power spectral density of $x(t), S_{x x}(\omega)$ can be expressed as:

$$
S_{x x}(\omega)=A_{\Upsilon}^{-1}(j \omega) \cdot S_{\xi \xi}(\omega) \cdot\left(A_{\Upsilon}^{-1}(j \omega)\right)^{*}
$$

The power spectral density of a random process describes the region in the frequency domain where power of the process exists. Thus, (10) describes how the input power is converted in the process by matrix $A_{\Upsilon}^{-1}(j \omega)$ to obtain the power content of $x(t)$, when the process disturbances and the inputs combined are supposed to be a wide sense stationary process. Therefore, $A_{\Upsilon}^{-1}(j \omega)$ gives information about the internal flow of energy in the process. The fact that $A_{\Upsilon}^{-1}(j \omega)$ is not a symmetric matrix involves that, the transmission from channel $\mathrm{i}$ to channel $\mathrm{j}$ is different than from channel $\mathrm{j}$ to channel $\mathrm{i}$, which means that $A_{\Upsilon}^{-1}(j \omega)$ is able to separate the energy flow in both directions.

Note that if the concept of DTF is applied to the MVAR process in (9), we obtain that $[D T F(\omega)]_{i j}^{2}=$ $\left[\Phi_{c}\left(A_{\Upsilon}^{-1}(j \omega)\right)\right]_{i j}$, and thus $\Phi_{c}\left(A_{\Upsilon}^{-1}(j \omega)\right)$ is a squared version of the DTF of the autoregressive part of the process. The main differences when using $\Phi_{c}\left(A_{\Upsilon}^{-1}(j \omega)\right)$ instead of DTF are:

- the normalization is performed in terms of the power transfer,

- the elements in the same column of $\Phi_{c}\left(\left(A_{\Upsilon}\right)^{-1}\right)$, add up to one.

$\Phi_{r}\left(A_{\Upsilon}^{-1}(j \omega)\right)$ is also proposed as a squared version of the DTF in which the comparison is made with respect to the sinks of the effect. We refer to those methods as squared DTF $(s D T F)$ :

$$
s D T F_{r}(\omega) \triangleq \Phi_{r}\left(A_{\Upsilon}^{-1}(j \omega)\right) ; s D T F_{c}(\omega) \triangleq \Phi_{c}\left(A_{\Upsilon}^{-1}(j \omega)\right)
$$

\section{E. Interpretation}

When a linear model of the process is available, the structural analysis of the plant described in Section III$\mathrm{C}$ gives a qualitative and quantitative description of the cause/effect relationships of the process around the working point.

If the propagation of the effects from the manipulated inputs and process disturbances is to be analyzed, the method in Section III-D.1 is applied. The columns of $F D P T_{c}$ and
$F E T_{c}$ compare the effects of a manipulated input or process disturbance in the process. The rows of $F D P T_{r}$ and $F E T_{r}$ compare the effects on a certain lumped state from the manipulated inputs and process disturbances.

In Section III-D.2 $s D T F_{r}$ and $s D T F_{c}$ are proposed as variants of the DTF used for measuring the internal propagation of power due to the interconnections of the process variables.

The following illustrative example is used in order to gain insight in the interpretation of the method.

\section{ILluStRATIVE EXAMPLE}

\section{A. Process description}

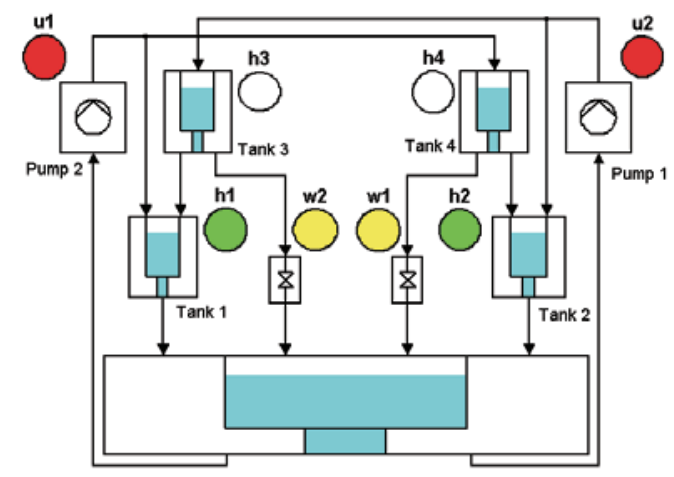

Fig. 2. Interacting system with water tanks.

The quadruple tank process described in [16], is depicted in Fig. 2. Two process disturbances $\left(w_{1}, w_{2}\right)$ have been added to the process, and modeled as a disturbance in the flow of tanks 3 and 4 with a maximum value of $1 \mathrm{~cm}^{3} / \mathrm{s}$. The modified differential equations describing the behavior of Tanks 3 and 4 are now:

$$
\begin{aligned}
& \frac{d h_{3}}{d t}=-\frac{a_{3}}{A_{3}} \sqrt{2 g h_{3}}+\frac{\left(1-\gamma_{2}\right) k_{2}}{A_{3}} v_{2}-\frac{1}{A_{3}} w_{1} \\
& \frac{d h_{4}}{d t}=-\frac{a_{4}}{A_{4}} \sqrt{2 g h_{4}}+\frac{\left(1-\gamma_{1}\right) k_{1}}{A_{4}} v_{1}-\frac{1}{A_{4}} w_{2}
\end{aligned}
$$

The physical values of the construction of the plant, and information on the selected working point $P_{-}$are given in [16]. When the process is linearized around $P_{-}$the following variables are introduced $\Delta h_{i}:=h_{i}-h_{i}^{0}$ and $\Delta u_{i}:=v_{i}-v_{i}^{0}$, where $h_{i}^{0}$ and $u_{i}^{0}$ denote the values at the working point.

\section{B. Analysis}

Matrix $a \Upsilon(s)$ for the selected working point is:

$$
a \Upsilon(s)=\left(\begin{array}{cc|cc}
0.0833 & 0 & 0 & 0 \\
0 & 0.0628 & 0 & 0 \\
0 & 0.0479 & 0.0357 & 0 \\
0.0357 & 0 & 0 & 0.0313
\end{array}\right)
$$

To gain insight in the meaning of these matrices we will have a look at the differential equations of the process. 
Take for instance, the first differential equation, which is describing the evolution of the variable $\Delta_{h_{1}}$,

$$
\begin{array}{r}
-0.0833 \Delta u_{1}(s)+(s+0.0159) \Delta h_{1}(s)-0.0419 \Delta h_{3}(s) \\
=\Delta h_{1}(0)
\end{array}
$$

the elements of the first row of matrix $a \Upsilon(s)$ in (12), are then the coefficients of the balance equation (13). Therefore, first row of $S D P T$ expresses the relative contribution of all the dynamic variables of the process into (13).

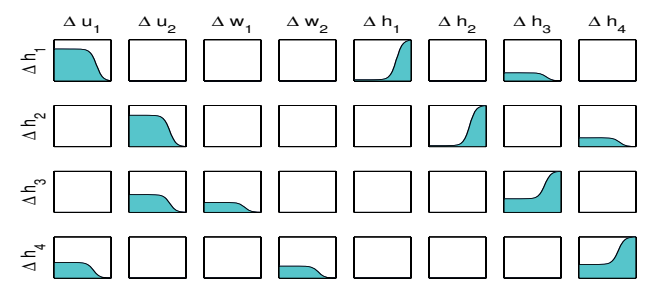

Fig. 3. SDPT for the quadruple tank process at the working point $P_{-}$for frequencies between $10^{-4} \mathrm{rad} / \mathrm{sec}$ and $10^{0} \mathrm{rad} / \mathrm{sec}$ (logarithmic scales). The ordinates axes are ranged between 0 and 1 (normalized).

As discussed in III-C, the fact that $a \Upsilon(s)$ is composed by non-proper transfer functions involves that the $\mathcal{H}_{2}$ norm of some its elements is not defined. In order to obtain an overall measure, matrix $\Upsilon(s)$ is computed:

$$
\begin{aligned}
\Upsilon(s)= & \left(\begin{array}{cc|cc}
\frac{0.0833}{s+0.0159} & 0 & 0 & 0 \\
0 & \frac{0.0628}{s+0.0111} & 0 & 0 \\
0 & \frac{0.00779}{s+0.0419} & \frac{0.0357}{s+0.0419} & 0 \\
\frac{0.0357}{s+0.0333} & 0 & 0 & \frac{0.0313}{s+0.0333}
\end{array}\right. \\
& \left(\begin{array}{cccc}
0 & 0 & \frac{0.0419}{s+0.0159} & 0 \\
0 & 0 & 0 & \frac{0.0333}{s+0.0111} \\
0 & 0 & 0 & 0 \\
0 & 0 & 0 & 0
\end{array}\right)
\end{aligned}
$$

Note that the main difference between matrix $\Upsilon(s)$ and matrix $a \Upsilon(s)$ is that the elements relating the lumped states with themselves have been set to 0 , and thus, the influence of the past values of a variable into its own future ones is not analyzed anymore. Take for example (13), and rearrange it to obtain:

$$
\begin{aligned}
\Delta h_{1}(s)=\frac{0.0833}{s+0.0159} \Delta u_{1}(s) & +\frac{0.0419}{s+0.0159} \Delta h_{3}(s) \\
& +\frac{1}{s+0.0159} \Delta h_{1}(0)
\end{aligned}
$$

The first row of matrix $\Upsilon(s)$ collects the coefficients of (14). Note that, the term $(s+0.0159)$ which is representing the own dynamic of $\Delta h_{1}$ is now lumped into the influence of the variables affecting it.

Applying $s \Sigma_{2, r}(\Upsilon(s))$, yields the overall measure $S E T$ (15), which determines the weights of the directed graph in Fig. 4.

$$
\begin{aligned}
& S E T= \\
& \left(\begin{array}{cccccccc}
0.8 & 0 & 0 & 0 & 0 & 0 & 0.2 & 0 \\
0 & 0.78 & 0 & 0 & 0 & 0 & 0 & 0.22 \\
0 & 0.64 & 0.36 & 0 & 0 & 0 & 0 & 0 \\
0.57 & 0 & 0 & 0.43 & 0 & 0 & 0 & 0
\end{array}\right)
\end{aligned}
$$

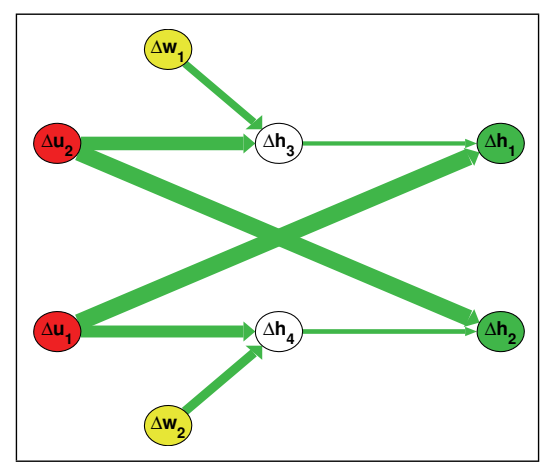

Fig. 4. Directed graph representing the Structural Energy Transfer (SET)

$\Upsilon(s)$ is in this case normalized from the point of view of the sinks of the effect, implying that the weights of all the edges entering a node in Fig. 4 add up to 1. $s \Sigma_{2, c}(\Upsilon(s))$ could also be applied. This would involve normalizing from the point of view of the sources of effect, which means that if a directed graph is generated from $s \Sigma_{2, c}(\Upsilon(s))$ the weights of all edges leaving a node would add up to one.

$F D P T_{r}$ and $s D T F_{r}$ are depicted in Fig. 5, and provide a description of the propagation of effects through the process, normalized from the point of view of the sources of the effect.

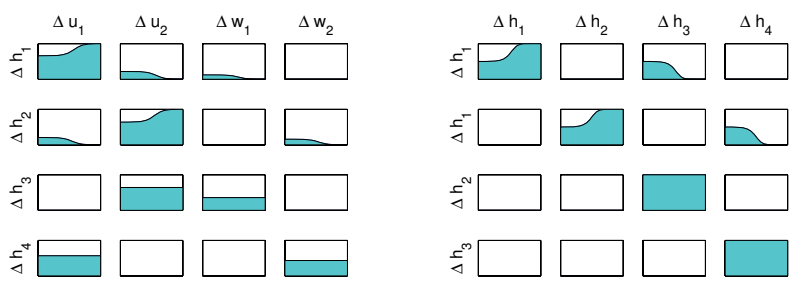

Fig. 5. FDPT $T_{r}$ (left) and $s D T F_{r}$ (right) for the quadruple tank process at the working point $P_{-}$for frequencies between $10^{-4} \mathrm{rad} / \mathrm{sec}$ and $10^{0} \mathrm{rad} / \mathrm{sec}$ (logarithmic scales). The ordinates axes are ranged between 0 and 1 (normalized).

$F D P T_{c}$ and $s D T F_{c}$ are depicted in Fig. 6, and provide a description of the propagation of effects through the process, normalized from the point of view of the sinks of the effect.

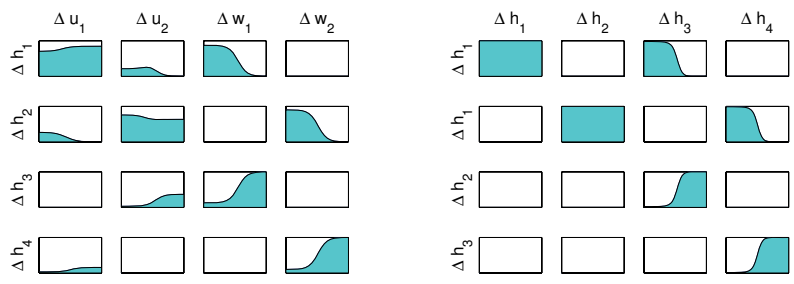

Fig. 6. $F D P T_{c}$ (left) and $s D T F_{c}$ (right) for the quadruple tank process at the working point $P_{-}$for frequencies between $10^{-4} \mathrm{rad} / \mathrm{sec}$ and $10^{0} \mathrm{rad} / \mathrm{sec}$ (logarithmic scales). The ordinates axes are ranged between 0 and 1 (normalized). 

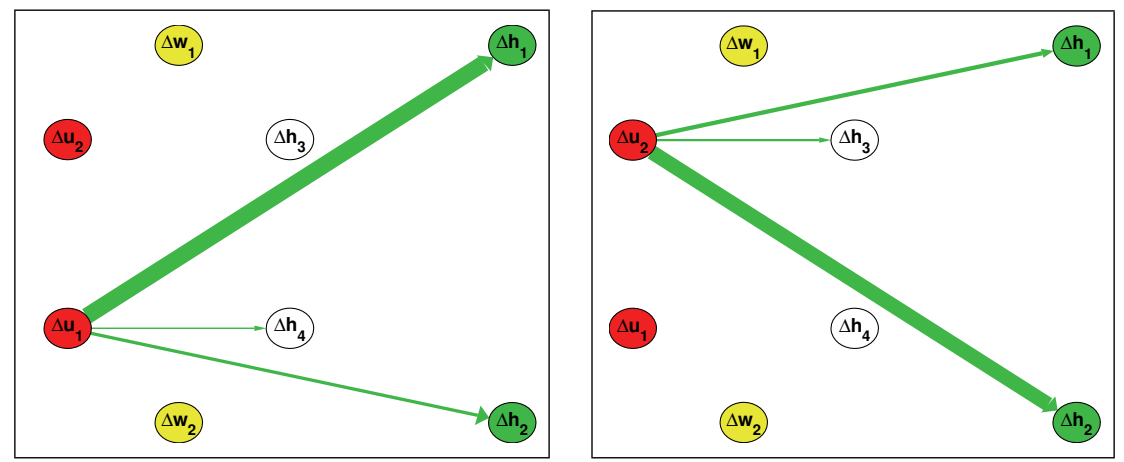

Fig. 7. Propagation of the effect from the inputs $\Delta u_{1}$ (left) and $\Delta u_{2}$ (right).
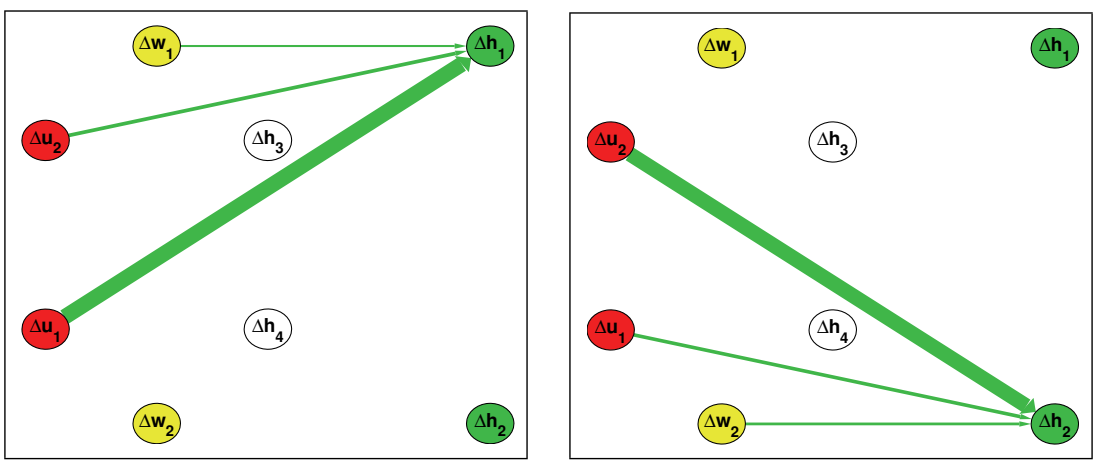

Fig. 8. Relative effect into the controlled variables $\Delta h_{1}$ (left) and $\Delta h_{2}$ (right) from the inputs and process disturbances

When overall measures are required to measure the propagation of the effect from manipulated inputs and process disturbances into the lumped states, $F E T_{r}$, and $F E T_{c}$ are computed.

$$
\begin{aligned}
F E T_{r} & =\left(\begin{array}{cccc}
0.73 & 0.17 & 0.1 & 0 \\
0.17 & 0.7 & 0 & 0.13 \\
0 & 0.64 & 0.36 & 0 \\
0.57 & 0 & 0 & 0.43
\end{array}\right) \\
F E T_{c} & =\left(\begin{array}{cccc}
0.78 & 0.2 & 0.66 & 0 \\
0.15 & 0.69 & 0 & 0.69 \\
0 & 0.11 & 0.34 & 0 \\
0.07 & 0 & 0 & 0.31
\end{array}\right)
\end{aligned}
$$

First and second columns of $F E T_{c}$ represent respectively overall measures of the relative effect of $\Delta u_{1}$ and $\Delta u_{2}$ into the lumped states. Both columns are used as weights for directed graphs depicted in Fig. 7.

Third and fourth column of $F E T_{c}$ represent the propagation of the effect of the process disturbances into the lumped states. They are used as weight for the directed graph depicted in Fig. 9.

First and second rows of $F E T_{r}$ represent the relative effect into the controlled variables from the inputs of the process. They are used as weight for the directed graph depicted in Fig. 8.

\section{Discussion}

$S E T$ in (15) and its associated graph in Fig. 4 provide a structural description of the process, allowing to visualize qualitative and quantitative information about the cause/effect relationship in the plant. When information is required in frequency domain, matrix $S D P T$ in Fig. 3 can be inspected. Inspecting either Fig. 4 or Fig. 3 we can deduce:

- Pump 1 is only directly affecting to the level of tanks 1 and 4 ,

- Pump 2 is only directly affecting to the level of tanks 2 and 3 ,

- the variation of level in Tank 3 is only affecting to the variation of level in Tank 1,

- the variation of level in Tank 4 is only affecting to the variation of level in Tank 2,

- process disturbance $w_{1}$ is directly affecting only to the level of Tank 3,

- process disturbance $w_{2}$ is directly affecting only to the level of Tank 4.

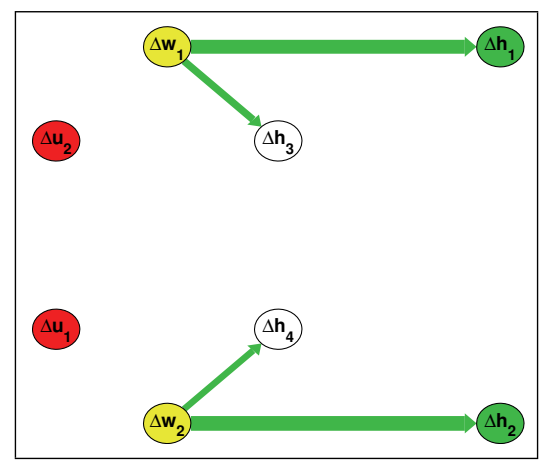

Fig. 9. Propagation of process disturbances. 
Hence the structure of the plant is described and direct effects compared, giving a first intuitive analysis of the process.

Nevertheless it can be noticed for example that Pump 1 also affects to the level of Tank 2, or that the process disturbance $w_{2}$ also affects to the level of Tank 2. Questions named in Section III-D then arise.

In order to answer these questions, different normalizations on matrix $\Omega(s)$ based on the concepts of $\Sigma_{2}$ and DTF have been proposed in Section III-D.

Fig. 7 shows the relative effect of the inputs into the lumped states. Regarding the controlled variables, it can be observed that, $\Delta u_{1}$ mainly affects to $\Delta h_{1}$ and $\Delta u_{2}$ mainly affects to $\Delta h_{2}$. An a priori decision about control structure is then to use the pairings $\Delta u_{1}-\Delta h_{1}$ and $\Delta u_{2}-\Delta h_{2}$ for decentralized control.

Fig. 8 shows the relative effect into the outputs from manipulated inputs and process disturbances. It can be concluded that $\Delta h_{1}$ is mainly affected by $\Delta u_{1}$ and $\Delta h_{2}$ is mainly affected by $\Delta u_{2}$, reinforcing the a priori decision about decentralized control.

Fig. 9 depicts the propagation of the process disturbances through the process. There it can be observed that even if $\Delta w_{1}$ is a variation of flow drained from Tank 3 the effect is higher into the level of Tank 1 . That perturbation implies the same loss of flow from the point of view of both tanks, which have similar construction, but the level at the working point is lower for Tank 3 which makes it being less sensitive to variations of flow. In the same way, the variable to which $\Delta w_{2}$ is affecting more is the level of Tank 2.

Even if the process disturbances $\Delta w_{1}, \Delta w_{2}$ are mainly affecting to the controlled variables $\Delta h_{1}, \Delta h_{2}$, inspecting Fig. 8 it can be observed that the effect of them is very low compared with the effect from the manipulated inputs selected for decentralized control. The analysis suggests that even the interaction received from the decentralized structure is expected to be larger than the effect of the process disturbances.

The internal propagation of energy between the states is represented by $s D T F_{r}$ (Fig. 5 (right)) and $s D T F_{c}$ (Fig. 6 (right)). When there is no internal feedback path, like in this case, the states can be reordered to a representation in which $s D T F r$ and $s D T F c$ are triangular. In the diagonal elements the relative importance of the own dynamics of the tanks can be observed, and in the off-diagonal elements the relative importance of the propagations of energy from Tank 3 to 1 and Tank 4 to 2 can be observed. The usefulness of $s D T F_{r}$ and $s D T F_{c}$ in industrial process analysis is still to be assessed, however, due to its resemblance to DTF, the reader can refer to the brain connectivity theory to gain insight about how DTF analyzes complex processes with feedback paths [13], [11] , [17].

\section{CONCLUSIONS}

Preliminary methods to describe the flow of energy in time-invariant processes linearized around a working point have been described. The methods are derived collecting and modifying elements used in interactions measures for control processes and in brain connectivity theory. The method is preliminary in the sense that it pretends to analyze the behavior of large scale industrial processes, whilst the introduced example has a very limited number of variables from which conclusions are easily derived.

The fact that the discussed methods depend on the scaling of the signals affects the interpretation of the results, and is focus of the further development of the methods.

\section{ACKNOWLEDGMENTS}

Funding provided by Swedish Governmental Agency for Innovation Systems (VINNOVA) and collaboration of the industrial partners of the SCOPE consortium are gratefully acknowledged.

The authors want to thank Jesús García and Pablo Fdez. de Dios for implementing these methods in a software tool for analysis of processes and control structure design.

\section{REFERENCES}

[1] M. Morari, Y. Arkun, and G. Stephanopoulos, "Studies in the synthesis of control structures for chemical processes. I. Formulation of the problem. process decomposition and the classification of the control schemes." AIChE J., vol. 26, no. 2, pp. 220-232, 1980.

[2] E. Bristol, "On a new measure of interaction for multivariable process control," IEEE Transactions on Automatic Control, vol. 11, pp. 133134, January 1966.

[3] A. Conley and M. E. Salgado, "Gramian based interaction measure," in Proc. of the 2000 Conference on Decision and Control, Sydney, 2000.

[4] B. Wittenmark and M. E. Salgado, "Hankel-norm based interaction measure for input-output pairing," in Proc. of the 2002 IFAC World Congress, Barcelona, 2002.

[5] W. Birk and A. Medvedev, "A note on gramian-based interaction measures," in Proc. of the European Control Conference 2003, University of Cambridge, UK, 2003.

[6] M. Morari and G. Stephanopoulos, "Studies in the synthesis of control structures for chemical processes. II. Structural aspects and the synthesis of alternative feasible control schemes," AIChE J., vol. 26, no. 2, pp. 232-246, 1980.

[7] H. Akaike, "On the use of a linear model for the identification of feedback systems," Annals of the Institute of Statistical Mathematics, vol. 20, pp. 425-439, December 1968.

[8] K. Fukunishi, "Coherence analysis of boiling water reactor noise," Journal of Nuclear Science and Thechnology, vol. 14, no. 5, pp. 351358, May 1977.

[9] R. Oguma, "Extended partial and multiple coherence analyses and their application to reactor noise investigation," Journal of Nuclear Science and Technology, vol. 19, pp. 543-554, July 1982.

[10] B. Halvarsson, "Comparison of some gramian based interaction measures," Computer-Aided Control Systems, 2008. CACSD 2008. IEEE International Conference on, pp. 138-143, Sept. 2008.

[11] L. A. Baccalá and K. Sameshima, "Partial directed coherence: a new concept in neural structure determination," Biological Cybernetics, no. 84, 463-474, 2001.

[12] L. Astolfi, F. Cincotti et al., "Assessing cortical functional connectivity by partial directed coherence: simulations and application to real data," pp. 1802-1812, Sept. 2006.

[13] M. Kamisnki and K. Blinowska, "A new method of the description of the information flow in the brain structures," Biological Cybernetics, 1991.

[14] T. Steffen, Control Reconfiguration of Dynamical Systems. Springer, 2005.

[15] M. Blanke et al., Diagnosis and Fault-Tolerant Control. Springer, 2003.

[16] K. Johansson, "The quadruple-tank process: a multivariable laboratory process with an adjustable zero," Control Systems Technology, IEEE Transactions on, vol. 8, no. 3, pp. 456-465, May 2000.

[17] A. Korzeniewska et al., "Determination of information flow direction among brain structures by a modified directed transfer function (ddtf) method," Journal of Neuroscience Methods, vol. 125, pp. 195-207. 